\title{
Educação nas redes sociais: o ensino do design e da moda através de páginas do Instagram
}

\author{
Isadora Franco Oliveira; \\ Glauber Soares Junior
}

resumo:

Este trabalho foi desenvolvido com o propósito de se compreender a importância das redes sociais no processo de ensino-aprendizagem, usando como contexto específico o caso de páginas do Instagram que são voltadas para o ensinamento de conceitos relativos ao design e a moda. Para tal, inicialmente foi desenvolvida uma pesquisa em fontes bibliográficas para deter de uma perspectiva geral acerca da temática estudada. Depois da consolidação das bases teóricas, foi feita uma busca no aplicativo citado, onde foram levantadas sete páginas distintas que possuem como foco o compartilhamento de informações técnicas e científicas relativas à temática do design e da moda. Quanto aos resultados, destaca-se que, através das funcionalidades disponibilizadas pelo Instagram, faz-se possível serem compartilhadas informações de maneira dinâmica e interativa, melhorando o diálogo existente entre o emissor e o recebedor da mensagem transmitida. Juntas, as páginas analisadas possuem mais de 31.000 seguidores, evidenciando a grande procura de interessados na temática através da rede social supracitada.

palavras-chave:

Educação; design; moda; Instagram 


\section{Introdução}

No mundo atual, a cada momento surgem inovações que transformam as relações sociais, e a internet é uma das grandes responsáveis por boa parte destas, gerando contribuições para variados setores da sociedade. Através do uso da internet, é propiciada a aquisição de informações de qualquer temática e lugar do mundo em poucos minutos. Nesse sentido, as redes sociais surgiram no ano de 2006, havendo a criação de inúmeros aplicativos e plataformas interativas. Desde sua criação, as redes sociais se tornaram parte da vida de muitas pessoas, sendo usadas para múltiplas finalidades (SILVA; SERAFIM, 2016).

$\mathrm{Na}$ contemporaneidade o volume e a circulação de informações são altos. A comunicação via redes sociais faz com que o processo de compartilhamento de informações seja intenso e rápido. Nesse sentido, através desse novo contexto social, novos processos educacionais são criados, traçando um diálogo do ensino-aprendizagem com essa nova realidade. Dentro desse contexto, faz-se essencial observar que, através dessa elevação de difusão de conhecimentos na internet - especificamente, nas redes sociais - tem-se intensificado também o cuidado pelo tipo de informação que está sendo fundada e compartilhada. Sendo assim, muitos autores (SILVA; MIRANDA, 2016; MEIRA, 2016; ARAUJO; OLINDA; PERALTA, 2017) vêm sugerindo a difusão de conhecimentos científicos nas redes sociais, evidenciando que essa prática pode gerar novos estudos e discussões (PEREIRA; SILVA JUNIOR; SILVA, 2019).

Desde o surgimento da internet, ocorreram diversas mudanças nas suas funções, no comportamento do usuário e das ferramentas disponíveis para uso. Com a evolução recente de plataformas como Instagram, Facebook, LinkedIn, dentre outras, o compartilhamento de informações e conteúdos se tornou mais fácil, ágil e interativo. Tais plataformas permitem uma maior colaboração entre os usuários, facilitando a disseminação do conteúdo (FAVERO; ALVAREZ, 2014).

O conteúdo criado para a rede tem o poder de auxiliar o desenvolvimento pessoal e profissional do usuário, se for de interesse do mesmo. As mídias sociais também podem aproximar marca e consumidor, instituições e produtos a usuários interessados, ou funcionar como uma plataforma de aprendizado (DAER; POTTS, 2014).

A possibilidade de troca de experiências promovida pela internet contribui para uma aprendizagem interativa, onde há uma comunicação maior, possibilitando que os papéis de aprender e ensinar sejam muitas vezes fundidos, não havendo uma demarcação clara de quem apenas ensina e quem apenas aprende. O campo de comentários e as mensagens diretas nos aplicativos e sites servem como uma plataforma para dúvidas e respostas, potencializando uma comunicação interpessoal (SILVA; MIRANDA, 2016).

A relação interpessoal é outro apelo das mídias sociais, onde os usuários são incentivados a compartilharem dos seus interesses, suas vidas e criarem de fato uma comunidade. Por essa razão, há um aumento da influência exercida entre pessoas de uma mesma comunidade. Isso significa que ao compartilhar uma postagem em seu próprio perfil, o usuário está endossando o conteúdo veiculado na mesma, divulgando-a para sua rede pessoal de amigos/seguidores, que estará mais inclinada a também consumir daquele conteúdo divulgado (FAVERO; ALVAREZ, 2014).

Dentro dessa perspectiva, este trabalho foi desenvolvido com intuito de compreender a importância das redes sociais no processo de ensino-aprendizagem, usando como contexto específico o caso de páginas do Instagram que são voltadas para o ensinamento de conceitos relativos ao design e a moda.

\subsection{Procedimentos metodológicos}

Com intuito de atingir o objetivo elencado, inicialmente o trabalho foi desenvolvido através de referências bibliográficas, intuindo possuir de uma perspectiva geral acerca da temática estudada. Segundo Marconi e Lakatos (2017), a pesquisa de fontes secundárias possibilita ao pesquisador um contato direto com tudo o que foi redigido sobre uma determinada temática. Para Gil (2008), a pesquisa bibliográfica é exigida em quase todos os estudos, sendo alguns compostos exclusivamente por revisão de literatura. Segundo o autor "a principal vantagem da pesquisa bibliográfica reside no 
fato de permitir ao investigador a cobertura de uma gama de fenômenos muito mais ampla do que aquela que poderia pesquisar diretamente" (GIL, 2018, p. 50).

Após a formalização das bases teóricas, o artigo foi direcionado através de um delineamento exploratório, com natureza qualitativa, em que foram selecionadas sete páginas da rede social Instagram que possuem como objetivo o compartilhamento de informações técnicas e científicas relativas ao design e moda. Nesse sentido, os dados dessa busca foram estudados por uma análise de conteúdo, como proposto por Bardin (2011).

\section{$2 \mathrm{~A}$ internet como plataforma de aprendizado}

A partir da criação de redes sociais (Facebook, Instagram, Twitter, entre outras), atualmente, muitas informações são apresentadas rapidamente e de maneira recorrente. Nesse contexto, tudo o que acontece no mundo pode ser visto, quase que instantaneamente na palma da mão, nas telas dos celulares. Sendo assim, as redes sociais exercem grande influência em seus participantes, que são majoritariamente adolescentes. (LIRA et al, 2017). É relevante dizer que o entendimento acerca do que são Redes Sociais precede o surgimento da tecnologia digital. Evidencia-se que a sociedade, por muito tempo vem sendo constituída por grupos sociais compostos por pessoas que possuem características homogêneas, formando suas comunidades específicas (SILVA; SERAFIM, 2016).

$\mathrm{Na}$ atualidade, as discussões relativas às redes sociais são pautadas através das redes digitais. Nesse contexto, alguns autores, como Silva e Serafim (2016), vêm analisando a relação dessas redes com o processo de aprendizagem. Nesse sentido, por redes sociais podem-se definir as plataformas que possuem os seguintes componentes: a) interação social; b) colaboração facilitada; e c) foco na deliberação entre usuários e membros, ou seja, as ações tomadas e as publicações feitas por usuários são de inteira responsabilidade dos mesmos (FEDOCK; MCCARTNEY; NEELEY, 2019).

$\mathrm{O}$ uso de redes sociais, inicialmente, era visto pelos seus usuários como uma maneira de se entreter, onde se buscavam conteúdos a fim de passar o tempo. Nos últimos anos tem-se identificado que muitas páginas criadas em tais redes (principalmente no Facebook e Instagram) surgem com intuitos distintos, tornando-se fontes de aquisição de conhecimentos e informação cientifica. Nesse contexto, muitas páginas que divulgam conteúdos de moda e design são vistas como locais de aprendizado, e muitas escolas e universidades vêm se adequando a essa nova realidade criando páginas e divulgando conteúdos intermediados pelos seus alunos. Santos (2020) salienta que, em decorrência da pandemia do covid-19, essas ferramentas foram consolidadas como um importante meio de comunicação entre professores e alunos.

De acordo com dados do IBGE, em 2017, 74,9\% dos domicílios brasileiros já possuíam acesso à internet. No mesmo levantamento, constatou-se que em $98,7 \%$ das casas que possuem acesso à internet, o celular é a principal fonte de acesso à mesma. Nesse sentido, Santos (2020) evidencia que o Facebook e o Instagram - de acordo com dados apresentados pelo próprio Facebook - juntos, possuem quase 200 milhões de contas ativas apenas no Brasil.

A criação de conteúdo na internet se tornou mais democrática com a disseminação das redes sociais. Muitos profissionais se utilizam dessas plataformas para compartilhar o que sabem sobre sua área, mostrando o dia-a-dia de suas profissões ou compartilhando recursos e conhecimentos que são úteis nas suas determinadas carreiras.

Coadunando com essa afirmativa, Meira (2016), diz que o uso de tecnologias e a velocidade no compartilhamento de informações são intrínsecos a sociedade contemporânea, ressaltando que se precisa cada vez mais investigar essa questão. Nessa perspectiva, a cada ano, novas ferramentas tecnológicas surgem, e essas possuem muitas alternativas de utilização. Sendo assim, essas inovações chegaram ao processo de ensino-aprendizagem. Evidencia-se então, que o uso dessas tecnologias pode gerar grandes impactos no ensino, já que, para os estudantes, a relação com o meio digital é tida como natural.

Para criar este tipo de conteúdo é imprescindível que o usuário já entenda a plataforma em que deseja criar sua comunidade. Ter uma conta e ser ativo na mesma é o primeiro passo para dar início ao compartilhamento de conteúdo autoral. É também interessante que o criador diversifique as plataformas em que disponibilizará o conteúdo, realizando uma curadoria de que tipo de postagem se encaixa melhor em cada plataforma. (DAER; POTTS, 2014) Um exemplo é a diferença entre uma postagem na plataforma Instagram, composta por uma foto e uma legenda com espaço limitado, 
versus uma postagem na plataforma YouTube, onde o formato de vídeo possibilita uma explicação mais detalhada do assunto abordado. Cada mídia e ferramenta possui diferentes recursos, implementações e audiências.

\subsection{0 ensino através das redes sociais}

$\mathrm{Na}$ atualidade, com o maior acesso a internet, as plataformas digitais se tornaram importantes formadoras de opinião. Nesse sentido, a internet é uma das principais fontes onde se buscam informações, principalmente pelo público mais jovem. Nessa perspectiva, é destacado que, as redes sociais como o Facebook e o Instagram influenciam no processo de ensino-aprendizagem, estimulando uma nova configuração de interatividade em relação à aquisição de conhecimentos. Dessa forma, precisa-se haver maior preocupação em serem difundidas informações com validade científica. (ARAUJO; OLINDA; PERALTA, 2017). Nesse aspecto:

Entende-se que o saber pedagógico, aquele referente ao uso, à finalidade, deve permear todo processo de articulação entre tecnologia e educação. Pois, [...] atualmente não se concebe pensar em sociedade sem a presença da tecnologia [...]. Assim, as tecnologias de informação e/ou comunicação possibilitam ao indivíduo ter acesso a uma ampla gama de informações e complexidades de um contexto (próximo ou distante) que, num processo educativo, pode servir como elemento de aprendizagem, como espaço de socialização, gerando saberes e conhecimentos científicos (SILVA; SERAFIM, 2016, p. 77).

Progressivamente, as redes sociais vão sendo modificadas e passam por um processo de evolução. Nessa perspectiva, atualmente, as mídias sociais possibilitam uma interatividade entre seus usuários em tempo real, e através do celular, um conteúdo pode ser compartilhado onde quer que a pessoa esteja. Sendo assim, a evolução de tecnologias móveis, possibilitou que o Instagram se tornasse uma das principais redes sociais existentes, revolucionando a maneira que fotografias e informações são partilhadas nos meios digitais (CAZORLA; NORONHA, 2015).

Segundo Moran, Seaman e Tinti-Kane (2011), algumas vantagens das mídias sociais facilitam o compartilhamento de conteúdos em suas plataformas, como a flexibilidade, seu caráter democrático e o fato de ser um conteúdo criado e controlado pelo usuário. A junção de todos esses aspectos define as redes sociais como uma plataforma descentralizada, onde informações se transformam em conversas, e estas podem provocar mudanças individuais, sociais e até mesmo globais.

Nessa circunstância, o uso de redes sociais no ensino é visto como algo de grande potencial, sendo necessário entender tais redes como formas de cultura e comunicação. Sendo assim, o avanço tecnológico está cada vez mais sendo efetivado em muitos setores sociais, fixando a sociedade cada vez mais em uma esfera digital (SILVA; SERAFIM, 2016).

Nesse contexto, essa digitalização da sociedade em termos de educação se intensificou em tempos de pandemia de covid-19, em que escolas e professores precisaram se adaptar ao uso de tecnologias no processo de ensino-aprendizagem, já que por um longo período, o distanciamento social tem sido visto como fundamental.

Portanto, a criação de conteúdo para plataformas digitais, garante ao aluno ou usuário a possibilidade de praticar o pensamento crítico ao selecionar os tópicos das postagens realizadas, além do aprimoramento de suas habilidades em transformar ideias em textos, imagens ou vídeos. Essa ação faz com que o aluno, em especial os mais jovens, ganhe interesse no assunto e até mesmo aumente sua vontade de aprender (GREENHOW; CHAPMAN, 2020). Nesse aspecto, estudos como o de Silva e Serafim (2016), evidenciam a relevância de se utilizar as novas tecnologias de informação, explorando suas potencialidades no campo educativo, ampliando por consequência a gama de opções de ferramentas que auxiliam o processo pedagógico contemporâneo. Nesse sentido, através do uso de tecnologias, as pessoas possuem acesso facilitado a inúmeras fontes informativas, que em um processo de aprendizagem, pode ser entendido como um local de socialização, propiciando a geração de conhecimentos científicos (SILVA; SERAFIM, 2016). 


\subsection{0 ensino do design e da moda através do Instagram}

Antes de se iniciar a discussão acerca do ensinamento do Design e da Moda através do Instagram, fazse fundamental, mesmo que de maneira sucinta, apresentar de que maneira se encontra o campo educacional do design e da moda no Brasil. As áreas citadas estão inclusas dentro de um mesmo campo de conhecimento e são regulamentados por uma mesma legislação. Nesse contexto, o ensino do Design possui um núcleo básico, possuindo variadas habilitações, sendo a Moda uma destas. Dessa forma, o "desenvolvimento de produtos é compreendido, sob a esfera do Design, como uma área de atuação abrangente que envolve saberes de domínios distintos" (PEREIRA; ANDRADE; MENEZES, 2015, p. 3).

Alguns autores (CAZORLA; NORONHA, 2015; SANTOS, 2020) vêm desenvolvendo trabalhos que refletem no aplicativo Instagram a característica de ser uma nova maneira de se divulgar conteúdos relacionados com a educação. Destaca-se que, através das redes sociais, são evidenciadas as vantagens do compartilhamento de informações, sendo algumas dessas, a fluidez e rápida movimentação das informações publicadas e o uso colaborativo, tornando a experiência interativa (CAZORLA; NORONHA, 2015).

O Instagram é um aplicativo que foi fundado no ano de 2010, sendo seu acesso e uso gratuitos. Tal aplicativo fornece aos seus usuários a viabilidade de fotografar, editar e publicar suas fotos em seus perfis. O aplicativo ainda possibilita o diálogo com outras redes sociais como Twitter e Facebook, em que podem ser compartilhados conteúdos que foram publicados inicialmente no Instagram. Nesse sentido, os recursos proporcionados pelo aplicativo (filtros, legendas, hashtags, entre outros), mediam a relação do aplicativo com o usuário (CAZORLA; NORONHA, 2015).

Nesse aspecto, o Instagram possui inúmeros recursos que podem e vêm sendo utilizados como maneiras de compartilhamento de informações pedagógicas. Destaca-se que no aplicativo são encontradas funciolanidades, tais quais: Feed: local onde são compartilhadas fotos dos usuários e vídeos que possuem até um minuto de duração; Stories: disponibilizado na tela inicial do Instagram, é um local onde são compartilhadas fotos e informações que possuem duração de 24 horas. Além disso, nos stories, tem-se a possibilidade de fazer perguntas que poderão ser respondidas por outras pessoas, tornando a ferramenta ainda mais interativa; Direct: local onde se podem enviar mensagens restritas a outro usuário; Live: ferramenta que possibilita a realização de transmissões em tempo real; IGTV: funcionalidade que dá aos seus usuários a possibilidade de vídeos mais longos; hashtags: são usadas para categorizar e agrupar temas que poderão ser buscados através de uma palavra precedida pelo símbolo cerquilha (\#) (SANTOS, 2020).

Sabendo das funcionalidades disponíveis no aplicativo citado, optou-se por fazer uma análise de 07 páginas do Instagram que possuem como finalidade o compartilhamento de informações voltadas para o ensinamento de conceitos de design e de moda. A escolha pela análise de perfis da plataforma Instagram se deu pelo fato de sua grande quantidade de acessos, que segundo o SEBRAE (201-) soma mais de um bilhão por mês, em todo o mundo, número em constante crescimento.

A comunicação interpessoal promovida pelas plataformas online possibilita o consumo e acesso a conteúdos específicos e de diferentes nichos, como é o caso dos perfis a seguir (quadro 1), analisados no presente trabalho quanto ao seu caráter divulgador de temas que abrangem o design e a moda.

Quadro 1 - Páginas analisadas

\begin{tabular}{|l|l|l|l|}
\hline Página & Local & Conteúdo & Seguidores \\
\hline $\begin{array}{l}\text { Projeto Moda.Collab } \\
\text { @moda.collab }\end{array}$ & $\begin{array}{l}\text { - Cultura de Moda } \\
\bullet \text { Design } \\
\text { IF Sudeste MG }-\end{array}$ & $\begin{array}{l}\text { Modelagem e Costura } \\
\text { Campus Muriaé } \\
\text { C Markultoria de Imagem } \\
\text { de Moda }\end{array}$ & 302 \\
\hline
\end{tabular}


Quadro 2 - Páginas analisadas (continuação)

\begin{tabular}{|l|l|l|l|}
\hline $\begin{array}{l}\text { Moda IFRN } \\
\text { @moda.ifrn }\end{array}$ & $\begin{array}{l}\text { IF Rio Grande do Norte } \\
\text { - Campus Caicó }\end{array}$ & $\begin{array}{l}\text { Modelagem } \\
\bullet \text { Técnicas de Desenho } \\
\bullet \text { Divulgação de } \\
\text { concursos e congressos }\end{array}$ & 594 \\
\hline $\begin{array}{l}\text { Design de Moda UniAcademia } \\
\text { @designdemodauniacademia }\end{array}$ & CES - Juiz de Fora, MG & $\begin{array}{l}\bullet \text { Divulgação e exposição } \\
\text { de trabalhos e criações } \\
\text { de alunos do curso }\end{array}$ & 1741 \\
\hline $\begin{array}{l}\text { História da Moda - UFJF } \\
\text { @historiadamoda.ufjf }\end{array}$ & $\begin{array}{l}\text { Universidade Federal de } \\
\text { Juiz de Fora }\end{array}$ & $\begin{array}{l}\bullet \text { História da arte } \\
\bullet \text { História da moda }\end{array}$ & 2590 \\
\hline $\begin{array}{l}\text { Moda sem Sacola } \\
\text { @modasemsacola }\end{array}$ & Juiz de Fora & $\begin{array}{l}\bullet \text { Slow Fashion } \\
\text { Moda consciente } \\
\bullet \text { História da Moda } \\
\bullet \text { Moda Artesanal }\end{array}$ & 2799 \\
\hline $\begin{array}{l}\text { Moda Limpa } \\
\text { @modalimpa }\end{array}$ & $\begin{array}{l}\text { São Paulo, SP/Salvador, } \\
\text { BA }\end{array}$ & $\begin{array}{l}\bullet \text { Plataforma com curso } \\
\text { sobre moda sustentável } \\
\bullet \text { Blog com tutoriais }\end{array}$ & 21.800 \\
\hline $\begin{array}{l}\text { Escola - Moda Online } \\
\text { @_modaonlinebr }\end{array}$ & $\begin{array}{l}\bullet \text { Negócios } \\
\bullet \text { Marketing de Moda } \\
\bullet \text { Pesquisa de Mercado }\end{array}$ & 1459 \\
\hline
\end{tabular}

Fonte: Autoria própria

Após o entendimento de que as redes sociais vêm sendo utilizadas como ferramentas de apoio pedagógicas, muitas páginas, essencialmente na plataforma do Instagram vêm sendo criadas com a finalidade de serem transmitidos conhecimentos técnicos e científicos. Nesse sentido, das sete páginas selecionadas para realização deste estudo, quatro são de origem universitária (Projeto Moda.Collab; Moda IFRN; Design de Moda UniAcademia; e História da Moda - UFJF) sendo estas mantidas por alunos e/ou docentes das respectivas instituições. Nessa conjuntura, em consonância com o que fora apontado pelos autores destacados no referencial teórico da pesquisa, em especial Cazorla \& Noronha (2015) e Santos (2020), através das funcionalidades disponibilizadas pelo aplicativo, faz-se possível serem compartilhadas informações de maneira dinâmica e interativa, melhorando o dialogo existente entre o emissor e o recebedor da mensagem transmitida.

Dentre as páginas analisadas, destaca-se o Projeto Moda.Collab (figura 1) desenvolvido e mantido por alunos do curso Superior de Tecnologia em Design de Moda do IF Sudeste MG, Campus Muriaé. Como destacado no quadro 1, os conteúdos da página abordam os temas: Cultura de Moda, Design, Modelagem e Costura, Consultoria de Imagem e Marketing e Consumo de Moda. A figura 1 corresponde a uma postagem realizada dentro da temática de design. Importante salientar que, o ensino da Moda no país é ainda muito recente. Destaca-se então que, no processo de ensino especifico da área, são apresentados conteúdos de Design, podendo ser compreendido que são ensinados aos estudantes de Moda conceitos do Design no desenvolvimento de seus produtos (PEREIRA; ANDRADE; MENEZES, 2015). 


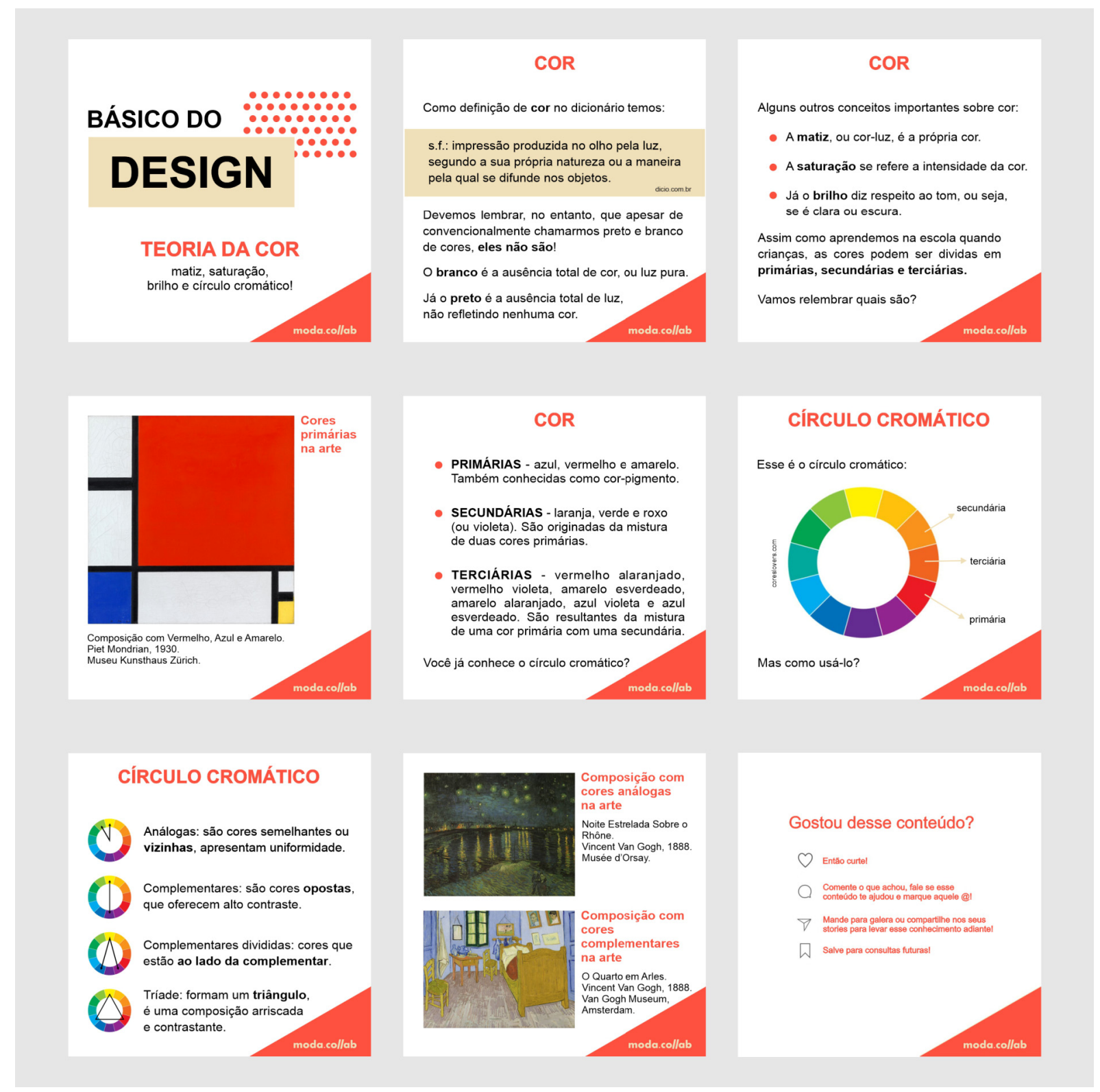

Figura 1 - Conteúdo da página @moda.collab

Fonte: Conteúdo disponível no endereço https://www.instagram.com/moda.collab com montagem realizada pelos autores

Em relação à página 'Moda IFRN', a mesma foi desenvolvida em março de 2020 com intuito de apresentar conteúdos relativos ao curso Superior de Tecnologia em Design de Moda do Instituto Federal do Rio Grande do Norte, ofertado pelo campus de Caicó. Nesse sentido, no decorrer das 61 publicações do perfil, dicas que versam a questões da moda, apresentadas por decentes e discentes. Ademais, o perfil também é utilizado como uma forma de divulgar eventos científicos que contemplam a área da moda.

Quanto ao conteúdo postado na página 'Design de Moda UniAcademia', nota-se que é uma forma de divulgação dos trabalhos dos alunos do curso da instituição juiz-forana. Com postagens sobre os trabalhos de conclusão de curso (TCC) de egressos, disseminação de eventos com transmissão ao vivo sobre temas relacionados à moda e eventos presenciais organizados pelo Centro Universitário UniAcademia (anteriormente chamado CES-JF), o perfil está no ar desde maio de 2012.

Como evidenciado em uma publicação do próprio perfil, 'História da Moda - UFJF', o mesmo foi desenvolvido em março de 2020, com intuito de divulgar as discussões e produções dos decentes e orientandos pertencentes ao Grupo de Pesquisa em História e Cultura de Moda da UFJF (Universidade Federal de Juiz de Fora). Até o momento da realização da pesquisa, a página possuía 115 publicações, 
das quais apresentam conteúdos relativos às áreas das artes e da moda, indicando livros e artigos, dissertações e teses que contemplam as temáticas citadas.

Com relação ao 'Moda Sem Sacola', criado em maio de 2017, é um perfil sobre moda consciente, com conteúdos relacionados a brechós, promoção de bazares, slow fashion (moda lenta) e moda artesanal. Ele complementa um site e uma revista sobre os mesmos temas, tratando ainda sobre sustentabilidade e consumo consciente.

A principal finalidade do perfil 'Moda Limpa' é a divulgação do próprio curso sobre moda, criado em julho de 2016 e realizado em São Paulo. Atualmente o curso é ofertado de forma online e tem como foco a moda sustentável. A página também possui conteúdos complementares ao curso, principalmente sobre tecidos, novas tecnologias têxteis, divulgação de marcas que seguem os conceitos de moda limpa e design sustentável.

O foco do perfil 'Escola - Moda Online' é em negócios e marketing de moda, possuindo também um podcast sobre o assunto. As postagens seguem a temática e abrangem conceitos como Design Thinking, Pesquisa de Mercado e Mapeamento de Persona, a fim de auxiliar os seguidores a entenderem melhor sobre como desenvolver uma marca de moda. Criada em abril de 2020, a página possuía 68 postagens até o momento de realização desta pesquisa.

Evidencia-se de maneira geral, que a maioria das páginas selecionadas para a análise teve o seu surgimento catalogado a partir do mês de março de 2020, sendo este o mês em que começaram a ser registrados, progressivamente casos do Covid-19 no país, acarretando no isolamento social de boa parte da população. Nesse sentido, coadunando com o pensamento de Malavé (2020), durante o isolamento social provocado pela pandemia, as redes sociais se tornaram ainda mais importantes para a sociedade em geral, produzindo uma nova potencialidade: ser um recurso no processo de ensino e aprendizagem.

\section{Considerações finais}

Com os adventos tecnológicos, a sociedade vem se remodelando a fim de se adaptar a nova realidade. Nesse sentido, através do uso de redes sociais no processo de ensino-aprendizado pode-se notar um aumento no número de perfis criados para esse fim. Das sete páginas analisadas nesta pesquisa, quatro foram criadas em 2020. Esse aumento se deve às oportunidades criadas pela tecnologia aplicada nas mídias sociais, onde é possível criar e receber conteúdo a todo tempo, a fim de gerar novas experiências de aprendizado que se estendem além da configuração de sala de aula. Pode-se entender ainda, que o aumento de páginas deste tipo nas redes sociais, tem ligação com o isolamento social causado pela epidemia do covid-19, já que as pessoas precisaram se adaptar a essa nova realidade. Quebrando a barreira geográfica, o conteúdo postado online atinge um número maior de indivíduos, criando uma nova comunidade entre seguidores interessados em um tema comum e, portanto, concebendo habilidades colaborativas no que tange o compartilhamento de conhecimento.

Nesse sentido, até a data de finalização da presente pesquisa, 31.285 pessoas seguiam os perfis selecionados para a realização da análise, evidenciando a grande procura de conteúdos técnicos e científicos relativos à área do design na rede social estudada. 
Education on social media: teaching design and fashion through Instagram profiles

\section{Abstract:}

This work was developed with the purpose of understanding the importance of social media in the teaching-learning process, where Instagram pages are a useful resource when it comes to teaching concepts related to design and fashion. To accomplish that, researches in bibliographical sources were initially developed to have a general perspective about the studied subject. After the consolidation of the theoretical base, search was conducted in the cited application, where the seven distinct pages that were picked focus on sharing technical and scientific information about design and fashion themes. The results highlighted that, through the functionalities made available by Instagram, it is possible to share information in a dynamic and interactive way, improving the existing dialogue between the sender and the receiver of the transmitted message. Together, the pages analyzed have more than 31,000 followers, showing the great demand of those interested in the subject through the aforementioned social media platform.

Keywords:

Education; design; fashion; Instagram

\section{Referências bibliográficas}

ARAUJO, A. Y. R. de; OLINDA, T. S; PERALTA, S. L. As redes sociais no processo de aprendizagem da disciplina de Dentística. In: CONEXÃO FAMETRO 2017: ARTE E CONHECIMENTO XIII SEMANA

ACADÊMICA, 8, 2017, Fortaleza. Anais [...]. [S.L.]: [S.N.], 2018. p. 1-6. Disponível em:

$<$ https://doity.com.br/media/doity/submissoes/artigo-fdffd88f9be087d5f2076f8c754f3d5c5f340b7farquivo.pdf>. Acesso em: 28 jul. 2020.

BARDIN, L. Análise de conteúdo. São Paulo: Edições 70, 2011.

CAZORLA, A. V; NORONHA, R. F. ALÉM DO LOOK DO DIA: o aplicativo Instagram e a moda contemporânea. In: ENPMODA - ENCONTRO NACIONAL DE PESQUISA EM MODA, 5., 2015, Novo

Hamburgo. Anais [...]. [S.L.]: [S.N.], 2015. p. 1-20. Disponível em:

<http://www.feevale.br/Comum/midias/5419ac29-bf8e-49e8-af26-

1eff083b32aa/AL\%C3\%89M\%20DO\%20LOOK\%20DO\%20DIA\%20-

\%20\%200\%20APLICATIVO\%20INSTAGRAM\%20E\%20A\%20MODA\%20CONTEMPOR\%C3\%82NEA.pd f>. Acesso em: 29 jul. 2020.

DAER, A. R.; POTTS, L. Teaching and Learning with Social Media: Tools, Cultures, and Best Practices, in: Programmatic Perspectives, vol. 6, n. 2, s. l:: 2014, p. 21-40. Disponível em:

<http://cptsc.org/wp-content/uploads/2018/04/vol6-2.pdf>. Acesso em: 26 jul. 2020.

FAVERO, M. B.; ALVAREZ, F. J. S. M. A Importância das Redes Sociais para o Setor Confecção:

Volume de Propagação da Mensagem, in: $\mathbf{1 0}^{\circ}$ Colóquio de Moda - 7a Edição Internacional e $1^{0}$

Congresso Brasileiro de Iniciação Científica em Design e Moda, s.l:: 2014. Disponível em:

$<$ http://www.coloquiomoda.com.br/coloquio2017/anais/anais/edicoes/10-Coloquio-de-

Moda_2014/COMUNICACAO-ORAL/CO-EIXO4-COMUNICACAO/CO-EIXO-4--A-importancia-das-redes-

sociais-para-o-setor-de-confeccao.pdf>. Acesso em: 25 jul. 2020.

FEDOCK, B. C.; MCCARTNEY, M.; NEELEY, D. Online adjunct higher education teachers' perception of using social media sites as instructional approaches, in: Journal of Research in Innovative

Teaching \& Learning, vol. 12, n. 3, p. 222-235. 2019. Disponível em:

<https://www.emerald.com/insight/content/doi/10.1108/JRIT-02-2018-0005/full/html>. Acesso em:

29 jul. 2020. 
GIL, A. C. Métodos e Técnicas de Pesquisa Social. 6. ed. São Paulo: Atlas, 2008. 220 p.

GREENHOW, C.; CHAPMAN, A. Social distancing meet social media: digital tools for connecting students, teachers, and citizens in an emergency, in: Information and Learning Sciences, vol. 121, n. 5/6, p. 331-342. 2020. Disponível em: <https://doi.org/10.1108/ILS-04-2020-0134>. Acesso em: 29 jul. 2020.

IBGE. Uso de Internet, televisão e celular no Brasil. Rio de Janeiro: [s. n.], 2017. Disponível em: <https://educa.ibge.gov.br/criancas/brasil/2697-ie-ibge-educa/jovens/materias-especiais/20787-usode-internet-televisao-e-celularnobrasil.html>. Acesso em: 21 jul. 2020.

LIRA, A. G. et al. Uso de redes sociais, influência da mídia e insatisfação com a imagem corporal de adolescentes brasileiras. Jornal Brasileiro de Psiquiatria. Rio de Janeiro, v. 66, n. 3, p. 164171, set. 2017. Disponível em: <http://www.scielo.br/scielo.php?script=sci_arttext\&pid=S0047$20852017000300164 \&$ Ing=en\&nrm=iso >. Acesso em: 29 jul. 2020.

MALAVÉ, M. M. O papel das redes sociais durante a pandemia. 2020. Instituto Nacional de Saúde da Mulher, da Criança e do Adolescente Fernandes Figueira (IFF/Fiocruz). Disponível em: <http://www.iff.fiocruz.br/index.php/8-noticias/675-papel-redes-sociais>. Acesso em: 30 jul. 2020.

MARCONI, M. A. d.; LAKATOS, E. M. Fundamentos de Metodologia Cientifica. 6. ed. São Paulo: Atlas S.A., 2017.

MEIRA, S. L. B. Redes Sociais Como Ferramenta de Ensino dos Fenômenos Ópticos. 2016. 136 f. Dissertação (Mestrado) - Curso de Mestrado Profissional de Ensino de Física, UNB, Brasília, 2016. Disponível em:

<http://mnpef.fis.unb.br/docs/dissertacoes/2016/samara_//dissertacao_samara.pdf>. Acesso em: 28 jul. 2020.

MORAN, M.; SEAMAN, J.; TINTI-KANE, H. Teaching, Learning, and Sharing: How Today's Higher Education Faculty Use Social Media, Pearson Learning Solutions. Boston, 2011. Disponível em:

<https://eric.ed.gov/?id=ED535130>. Acesso em: 27 jul. 2020.

PEREIRA, L. M; ANDRADE, R. R; MENEZES, M S. dos. AS ESPECIALIDADES DO DESIGN E O ENSINO DO DESIGN DE MODA. In: $11^{\circ}$ COLÓQUIO DE MODA - $8^{\circ}$ EDIÇÃO INTERNACIONAL $2^{\circ}$ CONGRESSO BRASILEIRO DE INICIAÇÃO CIENTÍFICA EM DESIGN E MODA, 11. 2015, Curitiba. Anais [...]. [S.L.]: [S.N.], 2015. p. 1-11. Disponível em:

<http://www.coloquiomoda.com.br/anais/Coloquio\%20de\%20Moda\%20-\%202015/COMUNICACAOORAL/CO-EIXO1-DESIGN/CO-1-AS-ESPECIALIDADES-DO-DESIGN-E-O-ENSINO-DO-DESIGN-DEMODA.pdf>. Acesso em: 30 jul. 2020.

PEREIRA, J. A; SILVA JÚNIOR, J. F. da; SILVA, E. V. da. Instagram Como Ferramenta de Aprendizagem no Ensino de Química. REDQUIM: Revista debates em ensino de química, s.l., v. 5, n. 1, p. 119-131, maio 2019. Disponível em:

<http://www.journals.ufrpe.br/index.php/REDEQUIM/article/view/2099/482483054>. Acesso em: 28 jul. 2020.

SANTOS, D. Muito além dos likes: como usar as redes sociais na educação. Nova Escola. 2020. Disponível em: <https://novaescola.org.br/conteudo/19124/muito-alem-dos-likes-como-usar-as-redessociais-no-ensino-a-distancia>. Acesso em: 29 jul. 2020.

SILVA, D. M. C.; MIRANDA, C. E. Aprendizado Colaborativo em Redes Sociais: Uma Reflexão Sobre as Possibilidades de Contribuição do Design, in: $\mathbf{1 2}^{\circ}$ Congresso Brasileiro de Pesquisa e

Desenvolvimento em Design, ed. 2, vol. 9, Belo Horizonte: 2016, p. 2190-2199. Disponível em: $<$ https://www.proceedings.blucher.com.br/article-details/aprendizado-colaborativo-em-redes-sociaisuma-reflexo-sobre-as-possibilidades-de-contribuio-do-design-24423>. Acesso em: 24 jul. 2020.

SILVA, F. S; SERAFIM, M. L. Redes sociais no processo de ensino e aprendizagem: com a palavra 0 adolescente. In: SOUSA, RP. et al, org. Teorias e práticas em tecnologias educacionais [online]. Campina Grande: EDUEPB, 2016, p. 67-98. ISBN 978-85-7879-326-5. Disponível em:

<http://books.scielo.org/id/fp86k/pdf/sousa-9788578793265-04.pdf>. Aceso em: 29 jul. 2020. 